\title{
Omalizumab in patients with NSAIDs-exacerbated respiratory disease*
}

\author{
Ulrike Förster-Ruhrmann 1,2, Dafni Stergioudi1,2, Greta Pierchalla1,2, \\ Joachim W. Fluhr ${ }^{3}$, Karl-Christian Bergmann ${ }^{3}$, Heidi Olze ${ }^{1,2}$ \\ ' Department of Oto-Rhino-Laryngology, Campus Virchow-Klinikum, Charité - Universitätsmedizin Berlin, Berlin, Germany \\ 2 Department of Oto-Rhino-Laryngology, Campus Mitte, Charité - Universitätsmedizin Berlin, Berlin, Germany \\ ${ }_{3}^{3}$ Clinic for Dermatology, Venerology and Allergy, Charité - Universitätsmedizin Berlin, Berlin, Germany
}

Rhinology 58: 3, 226 - 232, 2020

https://doi.org/10.4193/Rhin 19.318

*Received for publication:

September 12, 2019

Accepted: December 13, 2019

\begin{abstract}
Background: The association of acetylsalicylic acid (ASA) intolerance, chronic rhinosinusitis with nasal polyps (CRSwNP), asthma, or chronic urticaria is known as NSAID-exacerbated respiratory disease (N-ERD). N-ERD patients often suffer from recurrent nasal polyps, severe asthma or also from urticaria. The aim of the present study was to retrospectively analyze the clinical efficacy of anti-lgE antibody treatment with omalizumab in patients with confirmed N-ERD.
\end{abstract}

Methods: In the open trial with patients receiving verum patients with CRSWNP, confirmed N-ERD by oral or nasal ASA challenges, asthma or chronic urticaria were included in the study. Rhinological and pulmonary parameters were evaluated before and after 3, 6 and 9 months of therapy by rhinological questionnaires (CRS VAS-scores and RSOM-31), nasal polyp (NP)-, ACT-scores and FEV1 values. Urticaria activity was monitored clinically. N-ERD patients with aspirin desensitization were included as control group (follow-up 9 months).

Results: In the omalizumab group 16 patients were included (10 female, 6 male, mean age 51 yrs). CRS symptoms, RSOM-31- and NP-score decreased significantly following omalizumab therapy compared to baseline. The ACT-score (MV 16.5 to 20.6) and FEV1values (MV $80 \%$ to $89 \%$ ) improved significantly. No urticaria symptoms were reported after 3 months. Oral prednisolone therapy was required in one case and a repeated nasal sinus surgery in an additional case due to progression of NP. In the control group ( 8 female, 8 male, mean age $45 \mathrm{yrs}$ ) the NP-score was unchanged.

Conclusions: Omalizumab is an effective therapy option in N-ERD patients in a 9 month study period.

Key words: analgesic intolerance, CRSwNP, nasal polyps, N-ERD, omalizumab

\section{Introduction}

The association of acetylsalicylic acid (ASA) (Aspirin) intolerance, chronic rhinosinusitis with nasal polyps (CRSwNP), asthma or chronic urticaria is known as ASA intolerance syndrome. In 1902 three years after introduction of aspirin as therapy, Hirschberg (1) reported a patient with angioedema and breathlessness after ASA uptake. Further cases followed in $1922^{(2)}$ and $1967^{(3)}$. The disease is also known as "aspirin exacerbated respiratory disease" (AERD) or currently "non-steroidal anti-inflammatory drugs (NSAIDs)-exacerbated respiratory disease" (N-ERD) ${ }^{(4)}$. The prevalence of respiratory symptoms following NSAID intake have been reported by $1.8 \%$ of the general European population ${ }^{(4)}$. A meta-analysis shows a prevalence of $9.7 \%$ in patients with CRSwNP and $14.9 \%$ in patients with severe asthma ${ }^{(5)}$. The diagnosis of N-ERD is confirmed by oral, bronchial or nasal ASA challenge tests. In patients with severe asthma, nasal ASA challenges can be performed ${ }^{(4,6)}$. The pathomechanism of the $\mathrm{N}$-ERD is based on an altered arachidonic acid metabolism. Abnormal synthesis and secretion of eicosanoids leads to increased leukotrienes (LT), induced by inhibition of cyclooxygenase (COX) (7). In a former study we described the correlation of eicosanoid imbalances with the CRS symptoms nasal obstruction, rhinitis and smell impairment in patients with CRSwNP and N-ERD (8). Cytokines such as IL-5, IL-4 and IFN-gamma enhance the inflammatory processes, representing a mixed Th1/Th2 type of inflammation ${ }^{(9,10)}$. The role for staphylococcal enterotoxins 
(SAEs) acting as super antigens in the development of airway inflammation in N-ERD patients has also been suggested ${ }^{(11)}$.

Patients with N-ERD and CRSwNP undergo significantly more often surgeries due to recurrent nasal polyps than CRSwNP patients without N-ERD ${ }^{(12)}$. Asthma patients with N-ERD have significantly more emergency room visits with asthma exacerbations and need significantly more inhalative/oral corticosteroids than asthma patients without N-ERD disease ${ }^{(13)}$. Symptomatic drug therapy consists of topical glucocorticoids in CRSwNP in accordance with the EPOS 2012 guidelines ${ }^{(14)}$ and the asthma therapy in accordance with the Global Initiative for Asthma (GINA) ${ }^{(15)}$. Specific N-ERD therapies are ASA desensitization ${ }^{(16)}$ and leukotriene modifiers ${ }^{(4,17)}$. Oral corticosteroids, which often have to be used in severe cases of CRSwNP and asthma, are associated with a plethora of side effects ${ }^{(18)}$. Gevaert et al. described a significant improvement of severe CRSwNP after 16 weeks of therapy with anti-IgE monoclonal antibody omalizumab in 15 patients in a randomized, double-blind, placebo-controlled study. Interestingly, also 8 patients with N-ERD were included. However, ASA-provocations were not performed to confirm NERD ${ }^{(19)}$. Case studies of N-ERD patients treated with omalizumab were reported by Yalcin et al. ${ }^{(20)}$, and Bergmann et al. ${ }^{(21)}$. These authors did not analyze their patients systematically regarding validated rhinological and pulmonary parameters. The aim of the present study was to analyze the clinical efficacy of omalizumab in patients with confirmed N-ERD disease, CRSwNP, asthma and/ or chronic urticaria with validated outcome parameters.

\section{Material and methods}

\section{Patients}

Adult patients ( $\geq 18$ years) were included in this retrospective study at the ENT Clinic or the Allergy Center, Charité Berlin from 2012 to 2018 . The study was approved by the Charité ethics committee (EA 1/098/18/ EA 1/128/ 15). The diagnosis N-ERD was confirmed by oral or nasal ASA provocations ${ }^{(6,22,23)}$, CRS according to the EPOS 2012 criteria ${ }^{(14)}$, asthma according to the criteria of the Global Initiative for Asthma (GINA) ${ }^{(15)}$ and chronic urticaria according to the urticaria guidelines ${ }^{(24)}$.

Primarily rhinological retrospective data of confirmed N-ERD patients with aspirin desensitization were included as control group (following aspirin desensitization daily dosage of $300 \mathrm{mg}$ aspirin).

Patients of the omalizumab group and the aspirin desensitization group received mometasone furoate nasal spray $400 \mu \mathrm{g} /$ daily started at least 2 months before start of therapy and was not changed.

\section{Inclusion criteria}

Patients with chronic rhinosinusitis with nasal polyps (CRSwNP) and confirmed N-ERD (confirmation of N-ERD by nasal or oral
ASA challenge) and uncontrolled asthma or chronic urticaria were enrolled in the omalizumab group.

\section{Exclusion criteria}

Patients with immunosuppressive diseases, cystic fibrosis, pregnancy or lactation. Other biologics.

\section{Clinical findings}

Age, gender, clinical history, the number of nasal sinus surgeries, $\mathrm{N}$-ERD confirmed by nasal or oral ASA challenge ${ }^{(22,23)}$, rhinological, pulmonary, dermal symptoms and atopy status (serum total $\operatorname{lgE}$ and specific lgE levels to saisonal/ perennial aeroallergens according to the GA2LEN allergy diagnostic panel ${ }^{(25)}$, CAP FEIA system Phadia, Uppsala, Sweden) were evaluated in the omalizumab group.

Baseline and follow-up visits

Omalizumab group: Baseline visit and visits following 3, 6 and 9 months therapy;

Control group: Baseline visit and visits following 9 months of aspirin therapy.

\section{Rhinological parameters}

Evaluation of visual analogue scales (VAS) of the CRS-symptoms nasal obstruction, facial pain, anterior and posterior rhinitis, hyposmia ${ }^{(14)}$ and rhinosinositis-outcome-measurement-31 (RSOM31) questionnaire were performed ${ }^{(14)}$. Furthermore, the nasal polyp (NP)- score was assessed by nasal endoscopy (score 1: NP in middle meatus only; score 2: NP beyond middle meatus; score 3: NP not blocking the nose completely; score 4: NP completely obstructing the nose; sum of both nasal sides) ${ }^{(19)}$.

In the aspirin desensitization control group rhinological parameters (VAS-scores of the CRS-symptoms, RSOM-31- and the NP-scores) were performed.

\section{Pulmonary parameters}

The asthma control test (ACT)-score with ACT-score $\geq 20$ as controlled asthma; ACT-score $<20$ as uncontrolled asthma (26); and FEV1 values of spirometric measurements were assessed. Asthma medication levels according to Global Initiative for Asthma (GINA) score with steps 1-5 were recorded in the omalizumab group (15): GINA step 1: short-acting beta agonists (SABA), or low dose inhaled corticosteroids (ICS)-formoterol; step 2: low dose ICS, or low dose ICS-formoterol, or leukotriene receptor antagonist (LTR); step 3: low dose ICS-long-acting beta agonists (LABA), or medium dose ICS, or low dose ICS-LTRA, step 4: medium dose ICS-LABA, or high dose ICS, add-on tiotropium or LTRA; step 5: high dose ICS-LABA, add-on tiotropium, biologics or oral corticosteroids. For the analysis of asthma medication GINA levels before/ following omalizumab therapy omalizumab was excluded in GINA step 5 in this study. 
Table 1. Clinical parameters of N-ERD patients before omalizumab treatment.

Clinical parameters of N-ERD patients receiving omalizumab

\begin{tabular}{ll}
\hline $\begin{array}{l}\text { Sociographic parameters } \\
\text { Number of patients }\end{array}$ & 16 \\
$\begin{array}{l}\text { Age (years) } \\
\text { Gender }\end{array}$ & $\begin{array}{l}\text { MV } 50.7 \text { (Min 28; Max 65) } \\
\text { Phenotype }\end{array}$ \\
\hline N-ERD (\%) & $100 \%$ \\
CRSwNP (\%) & $100 \%$ \\
Asthma (\%) & $100 \%$ \\
Urticaria (\%) & $44 \%$ \\
\hline Laboratory parameters & \\
\hline $\begin{array}{l}\text { Total lgE (kU/l) } \\
\text { Positive specific lgE to aeroaller- } \\
\text { gens (\%) }\end{array}$ & MV 190.0 (Min 44; Max 505) \\
Eosinophils (\%) & $63 \%$ \\
\hline Rhinological parameters & MV $5.7 \%$ (Min 0.5; Max 12) \\
\hline Nasal sinus surgeries (number) & MV 5.6 (Min 1; Max 18) \\
Nasal polyp-score & MV 3.1 (Min 0 Max 4) \\
\hline Pneumological parameters & \\
\hline ACT-score & MV 16.5 (Min 10; Max 23) \\
FEV1 (\%) \\
GINA-steps
\end{tabular}

FEV1: forced expiratory volume in 1 second; GINA: Global Initiative for Asthma; Min: Minimum; Max: Maximum; MV: mean value; N-ERD: nonsteroidal anti-inflammatory drugs (NSAIDs)-exacerbated respiratory disease.

\section{Urticaria activity}

The outcome of urticarial symptoms was monitored as clinically absent or present in the omalizumab group.

\section{Therapy with omalizumab}

The dosis of omalizumab was administered according to serum total IgE-values and the weight of the patients according to standard protocol of manufacturer's instructions every 4 weeks (minimum dose $150 \mathrm{mg}$ s.c., maximum dose $600 \mathrm{mg}$ s.c.). The therapy of omalizumab required either uncontrolled asthma or chronic urticaria and was thus within the indication. Local and systemic effects were documented.

\section{Concomitant medication/ other procedure}

Other medical treatment during the study treatment period was observed. The asthma medication levels according to Global Initiative for Asthma (GINA) ${ }^{(15)}$ were recorded in the omalizumab group. As other procedure necessary nasal sinus operations due to progression of nasal polyps were recorded.

\section{Statistics}

Nonparametric tests (Friedman for more than two groups, Wilcoxon for pairwise comparison) were used for the comparison of the values of nasal polyps-, ACT-, RSOM-31- und VAS-scores ( $p$
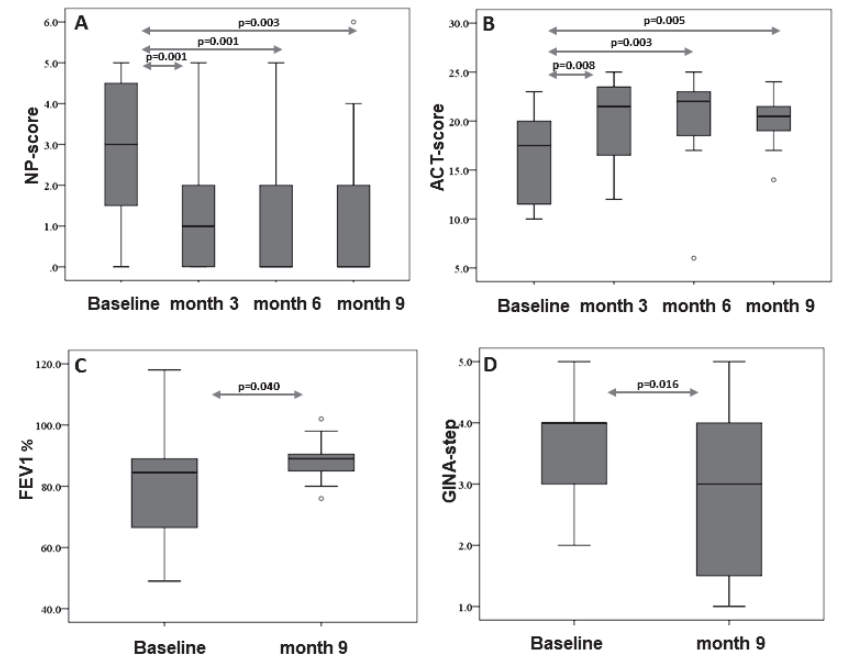

Figure 1. Clinical scores and FEV1 (\%) at baseline and following treatment with omalizumab. Panel A: nasal polyp-score, Panel B: ACT-score (baseline, following 3, 6 and 9 months); Panel C: FEV1 and Panel D: GINAstep (baseline and following 9 months). The respective $p$-values for pairwise comparison are depicted.

$<0.05)$. The baseline value was compared with the values at 3, 6 and 9 months of treatment, the baseline GINA- and FEV1-values were compared with the value at 9 months.

\section{Results}

\section{Characterization of $\mathrm{N}$-ERD patients}

In the omalizumab group 16 patients were included; 10 female and 6 male, with a mean age of 50.7 years (Min 28; Max 65). The N-ERD, CRSwNP and asthma was diagnosed in $100 \%$ and urticaria in $44 \%$ of all cases. Positive sensitizations to specific aeroallergens were diagnosed in 10 of 16 patients (63\%), Table 1. Analogue to the omalizumab group $16 \mathrm{~N}$-ERD patients ( 8 male, 8 female, mean age 45 years, Min 19; Max 65) were included in the aspirin desensitization control group (CRSwNP $100 \%$, asthma $100 \%$ with baseline MV FEV1 $85.2 \%$, no urticaria).

Nasal polyp score before and following medical treatment Following omalizumab therapy the nasal polyp score decreased significantly following 3, 6 and 9 months from initial MV 2.9 to MV 1.3 following 3 months ( $p=0.001$ ), MV 0.8 following 6 months ( $p=0.001)$ and MV 1.00 following 9 months ( $p=0.003)$ (Figure 1A).

Aspirin desensitization control group: Following 9 months of daily aspirin therapy the NP-score differed not significantly (MV 3.0 to $M V 2.9, p=0.75$.)

Pulmonary parameters before and following treatment with omalizumab

Before omalizumab therapy, an uncontrolled asthma was documented in $63 \%$ of cases $(10 / 16)$ which was reduced to $38 \%(6 / 16)$ following 9 months. The MV ACT-score improved 
Table 2. Mean p-values of VAS- and RSOM-31 scores before and following treatment with omalizumab.

\begin{tabular}{|c|c|c|c|c|c|c|c|}
\hline & Baseline & $\begin{array}{l}\text { Following } \\
3 \text { months }\end{array}$ & $\begin{array}{l}\text { Following } \\
6 \text { months }\end{array}$ & $\begin{array}{l}\text { Following } \\
9 \text { months }\end{array}$ & $\begin{array}{l}\text { p-value } \\
\text { Baseline/ } \\
3 \text { months }\end{array}$ & $\begin{array}{l}\text { p-value } \\
\text { Baseline/ } \\
6 \text { months }\end{array}$ & $\begin{array}{l}\text { p-value } \\
\text { Baseline/ } \\
9 \text { months }\end{array}$ \\
\hline VAS nasal obstruction & MV 59.6 & MV 35.1 & MV 39,8 & MV 34.7 & * & * & * \\
\hline VAS anterior rhinitis & MV 56.3 & MV 25.2 & MV 22.2 & MV 25.6 & $* *$ & $* * *$ & $* *$ \\
\hline VAS posterior rhinitis & MV 56.8 & MV 30.2 & MV 28.2 & MV 21.6 & * & $* *$ & $* * *$ \\
\hline VAS hyposmia & MV 86.3 & MV 62.2 & MV 63.3 & MV 65.9 & * & $* *$ & $* *$ \\
\hline VAS facial pain & MV 39.8 & MV 17.9 & MV 16.4 & MV13.4 & $* *$ & $* *$ & $* * *$ \\
\hline $\begin{array}{l}\text { RSOM-31 } \\
\text { Total score } \\
\text { Subdomains: }\end{array}$ & MV 7.8 & MV 4.1 & MV 3.9 & MV 3.6 & $* * *$ & $* * *$ & * \\
\hline -Nasal symptoms & MV 8.5 & MV 4.3 & MV 4.3 & MV 3.6 & $* * *$ & $* * * *$ & * \\
\hline -Ocular symptoms & MV 3.3 & MV 2.1 & MV 1.5 & MV 1.8 & $*$ & * & ns \\
\hline -Sleep & MV 8.8 & MV 5.4 & MV 5.4 & MV 6.0 & * & ns & ns \\
\hline -Ear symptoms & MV 3.2 & MV 1.4 & MV 1.8 & MV 1.4 & * & * & ns \\
\hline -General & MV 8.6 & MV 4.2 & MV 3.9 & MV 3.7 & $* *$ & $* * *$ & $* *$ \\
\hline -Practical problems & MV 9.1 & MV 4.8 & MV 4.0 & MV 2.8 & * & $* * *$ & $* *$ \\
\hline -Emotional consequences & MV 8.6 & MV 4.5 & MV 3.7 & MV 3.7 & $* *$ & $* *$ & ns \\
\hline
\end{tabular}

VAS: visual analogue scale; RSOM: rhinosinusitis outcome measurement; MV mean value; ${ }^{*}<0.05 ;{ }^{* *}<0.01 ;{ }^{* * *}<0.001$.

significantly from the baseline 16.5 points to 20.1 at month $3(p=0.008), 20.1$ at month $6(p=0.003)$ and 20.6 at month 9 $(p=0.005)$. The FEV1 mean value (\%) improved significantly following omalizumab therapy from $80 \%$ to $89 \%(p=0.040)$ (Figure 1B-1C).

\section{Rhinological questionnaires VAS-scores and RSOM-31}

The VAS-scores nasal obstruction, anterior and posterior rhinitis, hyposmia and facial pain improved significantly following 3, 6 and 9 months of omalizumab therapy. The RSOM-31 total score, the subdomains nasal symptoms, general, and practical problems decreased significantly following 3, 6 and 9 months compared to the pre-treatment status. Ear-/ ocular problems and emotional consequences decreased significantly following 3 and 6 months, sleep problems decreased significantly following 3 months (Table 2). Aspirin desensitization control group: The VAS-scores nasal obstruction (MV 43.5 to 19.7, $\mathrm{p}=0.034$ ) and anterior rhinitis (MV 38.1 to $14.6, p=0.044$ ) decreased significantly, following 9 months whereas the posterior rhinitis, hyposmia and facial pain differed not significantly. The total RSOM-31 total score decreased not significantly (MV 117 to $62.2, p=0.074$ ).

\section{Urticaria activity}

In all 7 patients with chronic urticaria symptoms were completely controlled during the 9 months of omalizumab therapy.

Results in the omalizumab group according sensitization to specific aeroallergens

From baseline to follow-up of 9 months the NP-score decreased not significantly in the group without sensitization to specific aeroallergens (NP-score MV 3.3 to 1.7; $\mathrm{p}=0.125$ ) and significantly in the group with positive sensitization (NP-score MV 2.6 to MV $0.6 ; p=0.031$ ), more details are given in Table 3 .

\section{Concomitant medication/ other procedure}

The urticaria treatment of high dosage levocetirine (up to $4 \mathrm{tbl}$ per day) was stopped after 3 symptom free months. The asthma medication decreased significantly from GINA-step MV 3.8 to 3.0 ( $p=0.016$ ) after 9 months of omalizumab therapy (Figure 1D). Details of asthma medication are given in Figure 2. Before omalizumab therapy oral prednisolone was needed in 3 cases ( 2 $x 5 \mathrm{mg}, 1 \times 20 \mathrm{mg}$ prednisolone per day) regarding pulmonary treatment, following 9 months in 2 cases $(1 \times 5 \mathrm{mg}, 1 \times 10 \mathrm{mg}$ prednisolone per day). No patient received oral prednisolone regarding nasal sinus problems before start of omalizumab therapy. At the end of the omalizumab therapy (9 months), one patient needed oral prednisolone and another patient needed a repeated nasal sinus surgery because of a progression of nasal polyps. No additional medication (e.g. oral prednisolone) was given in the aspirin desensitization control group.

\section{Safety aspects}

The omalizumab therapy was well tolerated. In one patient, local skin redness measuring about $3 \mathrm{~cm}$ in diameter occurred directly after the injections after 4 and 8 weeks, persisting approximately 2 hours. There were no systematic reactions. In the aspirin desensitization control group no adverse event was observed. 
Table 3. Mean p-values of NP-, ACT-, VAS-, RSOM-31-scores, FEV1- and GINA-values before and following 9 months of omalizumab treatment in subgroups without $(n=6)$ and with sensitization to specific aeroallergens $(n=10)$.

\begin{tabular}{|c|c|c|c|c|c|c|}
\hline & \multicolumn{6}{|c|}{ Baseline versus 9 months omalizumab treatment } \\
\hline & \multicolumn{3}{|c|}{ No sensitization to aroallergens $(n=6)$} & \multicolumn{3}{|c|}{ Positive sensitization to aeroallergens $(n=10)$} \\
\hline & Baseline & $9 \mathrm{mo}$ & p-value & Baseline & $9 \mathrm{mo}$ & p-value \\
\hline NP-score & MV 3.3 & MV 1.7 & ns & MV 2.6 & MV 0.6 & * \\
\hline ACT-score & MV 17.7 & MV 21.8 & * & MV 15.8 & MV 19.0 & ns \\
\hline FEV1 \% & MV 89.0 & MV 91.2 & ns & MV 74.6 & MV 87.1 & * \\
\hline GINA-step & MV 3.2 & MV 2.3 & ns & MV 4.1 & MV 3.4 & ns \\
\hline VAS nasal obstruction & MV 68.3 & MV 29.2 & * & MV 54.3 & MV 38.0 & ns \\
\hline VAS anterior rhinitis & MV 65.0 & MV 21.7 & * & MV 51.0 & MV 28.0 & ns \\
\hline VAS posterior rhinitis & MV 55.8 & MV 12.5 & * & MV 57.3 & MV 27.0 & * \\
\hline VAS hyposmia & MV 80.0 & MV 56.7 & ns & MV 90.0 & MV 71.5 & ns \\
\hline VAS facial pain & MV 49.2 & MV 6.7 & * & MV 34.2 & MV 17.5 & * \\
\hline RSOM-31 Total score & MV 7.2 & MV 2.5 & * & MV 8.1 & MV 4.2 & * \\
\hline
\end{tabular}

VAS: visual analogue scale; RSOM: rhinosinusitis outcome measurement; MV mean value; ${ }^{*}<0.05 ;{ }^{* *}<0.01 ;{ }^{* * *}<0.001$

\section{Discussion}

To our knowledge, this is the largest group with confirmed $\mathrm{N}$-ERD treated with omalizumab with the longest follow-up published so far. We could show that the rhinological and pulmonary parameters improved significantly following 3, 6 and 9 months of omalizumab therapy compared to baseline. The nasal polyp scores decreased significantly under omalizumab therapy. The VAS on CRS symptoms and the total RSOM-31 symptom score decreased significantly following 3, 6 and 9 months of omalizumab therapy. The subdomains of the RSOM-31 questionnaire showed a significant improvement for general and practical problems e.g. the need to use of paper towels indicating an improvement of quality of life.

The N-ERD disease is associated with severe progression of nasal polyps and asthma. New treatment options with biologics in

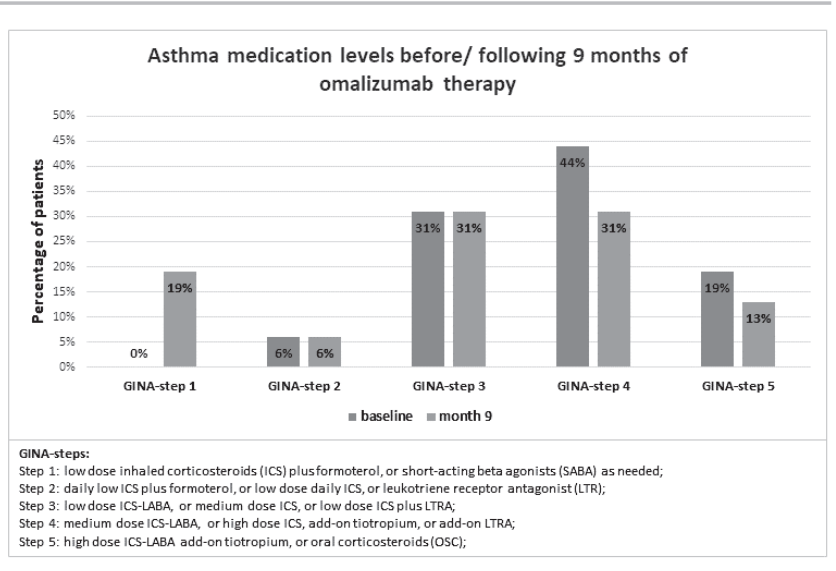

Figure 2. Global Initiative for Asthma (GINA) asthma medication levels at baseline and following 9 months treatment with omalizumab. Before omalizumab therapy oral prednisolone (GINA-step 5) was needed in 3 cases (19\%), following 9 months in 2 cases (13\%).
N-ERD disease are of high clinical interest ${ }^{(27)}$. Omalizumab prevents the binding of $\lg E$ to $\lg E$ receptors on mast cells and other effector cells, reduces free lgE and the number of high-affine lgE receptors on basophils ${ }^{(28)}$. Gevaert et al. treated patients with severe nasal polyps and comorbid asthma with omalizumab ${ }^{(19)}$. They showed a significant reduction of nasal polyps following therapy with omalizumab in allergic and in nonallergic nasal polyps, which we could confirm with relatively small subgroups only in the group of patients with positive sensitization to specific aeroallergens. Local IgE in nasal polyps was shown to be functional and able to activate mast cells independently of the presence of IgE in serum ${ }^{(29)}$. This may explain the positive effects of omalizumab in nonallergic patients with nasal polyps and negative prick test in the study of Gevaert ${ }^{(19)}$. N-ERD is characterized by non-lgE mediated intolerance reactions to aspirin and a treatment with an anti-lgE antibody is proven for IgE-mediated allergic patients. Gevaert et al. included ASA-intolerant and ASAtolerant patients, but the patients were not challenged with ASA prior to inclusion. In our study the control group consisted of proved N-ERD patients with aspirin desensitization. During the following 9 months of aspirin therapy no significant reduction of nasal polyps was observed. In the European position paper of $\mathrm{N}$-ERD aspirin desensitizations are recommended ${ }^{(4)}$, the relevance of the aspirin desensitization compared to biological treatment has to be investigated in further studies.

Szczeklik et al. conducted a multicenter study on 500 asthma patients with suspected N-ERD and reported that $15 \%$ of patients were unaware of N-ERD before oral or bronchial ASA challenges, underscoring the importance of ASA challenges to accurately describe the phenotype N-ERD ${ }^{(30)}$. In asthma patients, the efficacy of omalizumab therapy is widely recognized ${ }^{(31)}$ and is recommended in the GINA guidelines for severe asthma with 
persistent allergies (GINA). In our study the pulmonary parameters ACT-score, FEV1 and asthma medication levels improved significantly following omalizumab therapy. Yalcin et al. treated 3 patients with persistent allergies, severe asthma, CRSwNP and N-ERD with omalizumab for 32 weeks ${ }^{(20)}$. An ASA challenge was not described. The pulmonary parameters FEV1 and the ACT-score improved, but the nasal polyps were not assessed endoscopically in their study. Bergmann et al. reported the first patient with a confirmed N-ERD disease and concomitant severe asthma and a CRSwNP treated with omalizumab. The diagnosis $\mathrm{N}$-ERD was confirmed with an oral ASA challenge three weeks following the first omalizumab injection. The pulmonary parameters showed a good response to omalizumab. Interestingly, no perennial allergies were detectable in this case report (21). In the following studies also patients with severe asthma were examined, Bidder et al. ${ }^{(32)}$ examined patients with severe asthma and CRSwNP and compared a patient group receiving omalizumab ( $n=13$ ) with a patient group receiving nasal sinus operation $(n=24)$ before therapy and following 4 and 16 weeks. In both groups also N-ERD patients were included, in the omalizumab group $77 \%$ (10/13) and in the operation group $63 \%(15 / 24)$. The rhinological and pulmonary symptoms evaluated by SNOT and asthma control questionnaires ACQ-7 improved in both groups significantly following 4 and 16 weeks. The improvement of nasal polyp size following both therapy concepts was similar. But, also in this study ASA challenges were not performed. Tiotiu et al. reported 24 patients with also severe asthma and nasal polyps treated with omalizumab, 15 ASA tolerant patients and 9 ASA intolerant patients ${ }^{(33)}$. It is unclear if the group of ASA tolerant patients were challenged with ASA to exclude the N-ERD disease. Similar to our results the CRS symptoms loss of smell, nasal obstruction, and rhinorrhea significantly decreased following 6 months of omalizumab therapy, but nasal polyps did not improve. The authors considered the different ENT investigators of the nine N-ERD patients in the multicentric study as a possible reason for the negative result in this mainly pulmonary study. In addition to rhinological and pulmonary response in the present study, we showed a response to chronic urticaria in N-ERD patients. To our knowledge, only one case report of a young patient with a CRSwNP, severe asthma and chronic urticaria has been described in the literature ${ }^{(34)}$. During 6 months of therapy with omalizumab, an improvement of respiratory and urticarial symptoms was reported in this case report. However, no ASA challenge was performed.

In two N-ERD patients of our current study, the benefit for CRSwNP disease was insufficient following omalizumab therapy regarding the progress of nasal polyps. This highlights, in addition to the precise definition of the N-ERD phenotype, the need to determine additionally the endotype of patients. The endotyping primarily describes inflammatory pattern, published by Tomassen et al., which was secondarily assigned to the phenotypes of CRSWNP ${ }^{(35)}$. Additional predictive inflammatory parameters from nasal secretion, blood or nasal polyp biopsies could improve the choice of antibodies for personalized therapy of N-ERD patients ${ }^{(27)}$. Recently recommendations for the use of biologica in severe nasal polyps were given ${ }^{(36)}$ and significant reduction of nasal polyps was reported following therapy with dupilumab in CRSwNP (37) and also in N-ERD patients ${ }^{(38)}$.

\section{Conclusion}

In conclusion, our study could show that omalizumab is an effective therapy for rhinological, pulmological parameters, and urticaria in N-ERD patients. Larger, prospective, controlled multicenter studies are required to establish a treatment regimen with omalizumab in N-ERD.

\section{Acknowledgements}

Not applicable.

\section{Authorship contribution}

UFR: designed and coordinated the study, participated in the data acquisition and analysis, interpreted the data and drafted the manuscript. DS: participated in the data acquisition and analysis, interpreted the data and drafted the manuscript; GP: participated in the data acquisition and analysis, interpreted the data and drafted the manuscript; JWF: participated in the data analysis, interpreted the data and critically drafted the manuscript; KCB: participated in the data acquisition, interpreted the data and critically drafted the manuscript. HO: designed the study, critically interpreted the data and critically drafted the manuscript. All authors made substantial contributions to the study.

\section{Conflict of interest}

None related to this study.

\section{References}

1. Hirschberg. Anaphylactoid reaction to aspirin (1902). Allergy Proc. 1990;11(5):249-50; discussion 51-2.

2. Widal F, Abrami P, Lermoyez J. First complete description of the aspirin idiosyncrasy-asthma-nasal polyposis syndrome (plus urticaria)--1922 (with a note on aspirin desensitization). By F. Widal, P. Abrami, J. Lermoyez. J Asthma. 1987;24(5):297-300.

3. Samter M, Beers RF, Jr. Intolerance to aspirin. Clinical studies and consideration of its pathogenesis. Ann Intern Med. 1968;68(5):975-83.

4. Kowalski ML, Agache I, Bavbek S, Bakirtas A, Blanca M, Bochenek G, et al. Diagnosis and management of NSAID-Exacerbated Respiratory Disease (N-ERD)-a EAACI position paper. Allergy. 2019;74(1):28-39.

5. Rajan JP, Wineinger NE, Stevenson DD, White AA. Prevalence of aspirin-exacerbated respiratory disease among asthmatic patients: A meta-analysis of the literature. J Allergy Clin Immunol. 2015;135(3):676-81. 
6. Forster-Ruhrmann U, Tietz A, Kim J, Liebers U, Szczepek A, Olze H. Can the severity of N-ERD be predicted by nasal challenge with acetylslicylic acid ? Allergologie. 2018;12:551-559.

7. Szczeklik A, Sanak M. The broken balance in aspirin hypersensitivity. Eur J Pharmacol. 2006;533(1-3):145-55.

8. Forster U, Strathmann S, Schafer D, Szczepek AJ, Olze H. Eicosanoid imbalance correlates in vitro with the pattern of clinical symptoms of Samter's triad. Rhinology. 2013;51(1):61-9.

9. Perez-Novo CA, Watelet JB, Claeys C, Van Cauwenberge $\mathrm{P}$, Bachert C. Prostaglandin leukotriene, and lipoxin balance in chronic rhinosinusitis with and without nasal polyposis. J Allergy Clin Immunol. 2005;115(6):1189-96.

10. Steinke JW, Liu L, Huyett P, Negri J, Payne SC, Borish L. Prominent role of IFN-gamma in patients with aspirin-exacerbated respiratory disease. J Allergy Clin Immunol. 2013;132(4):856-65 e1-3.

11. Corriveau MN, Zhang N, Holtappels G, Van Roy N, Bachert C. Detection of Staphylococcus aureus in nasal tissue with peptide nucleic acid-fluorescence in situ hybridization. Am J Rhinol Allergy. 2009;23(5):461-5.

12. Van Zele T, Holtappels G, Gevaert P, Bachert C. Differences in initial immunoprofiles between recurrent and nonrecurrent chronic rhinosinusitis with nasal polyps. Am J Rhinol Allergy. 2014;28(3):192-8.

13. Mascia K, Haselkorn T, Deniz YM, Miller DP, Bleecker ER, Borish L, et al. Aspirin sensitivity and severity of asthma: evidence for irreversible airway obstruction in patients with severe or difficult-to-treat asthma. J Allergy Clin Immunol. 2005;116(5):970-5.

14. Fokkens WJ, Lund VJ, Mullol J, Bachert C, Alobid I, Baroody F, et al. EPOS 2012: European position paper on rhinosinusitis and nasal polyps 2012. A summary for otorhinolaryngologists. Rhinology. 2012;50(1):1-12

15. Reddel HK, FitzGerald JM, Bateman ED Bacharier LB, Becker A, Brusselle G, et al GINA 2019: a fundamental change in asthma management: Treatment of asthma with short-acting bronchodilators alone is no longer recommended for adults and adolescents. Eur Respir J. 2019;53(6).

16. Zeiss CR, Lockey RF. Refractory period to aspirin in a patient with aspirininduced asthma. J Allergy Clin Immunol. 1976;57(5):440-8

17. Grundmann T, Topfner M. [Treatment of ASS-Associated Polyposis (ASSAP) with a cysteinyl leukotriene receptor antagonist - a prospective drug study on its antiinflammatory effects]. Laryngorhinootologie. 2001;80(10):576-82

18. Al Efraij K, Johnson KM, Wiebe D, Sadatsafavi M, FitzGerald JM. A systematic review of the adverse events and economic impact associated with oral corticosteroids in asthma. J Asthma. 2018:1-13.

19. Gevaert P, Calus L, Van Zele T, Blomme K, De Ruyck N, Bauters W, et al. Omalizumab is effective in allergic and nonallergic patients with nasal polyps and asthma. J Allergy Clin Immunol. 2013;131(1):110-6 e1.

20. Yalcin AD, Ucar S, Gumuslu S, Strauss LG. Effects of omalizumab on eosinophil cationic peptide, 25-hydroxyvitamin-D, IL-1 beta and SCD200 in cases of Samter's syndrome: 36 months follow-up. Immunopharmacol Immunotoxicol. 2013;35(4):524-7.

21. Bergmann KC, Zuberbier T, Church MK. Omalizumab in the treatment of aspirinexacerbated respiratory disease. J Allergy Clin Immunol Pract. 2015;3(3):459-60.

22. Nizankowska-Mogilnicka E, Bochenek G, Mastalerz L, Swierczynska M, Picado C, Scadding G, et al. EAACI/GA2LEN guideline: aspirin provocation tests for diagnosis of aspirin hypersensitivity. Allergy. 2007;62(10):1111-8

23. Riechelmann $H$, Bachert C, Goldschmidt O, Hauswald B, Klimek L, Schlenter WW et al. [Application of the nasal provocation test on diseases of the upper airways. Position paper of the German Society for Allergology and Clinical Immunology (ENT Section) in cooperation with the Working Team for Clinical Immunology]. Laryngorhinootologie. 2003;82(3):183-8.

24. Zuberbier $T$, Aberer W, Asero R, Abdu Latiff AH, Baker D, Ballmer-Weber B, et al. The EAACI/GA(2)LEN/EDF/WAO guideline for the definition, classification, diagnosis and management of urticaria. Allergy. 2018;73(7):1393-414.

25. Bousquet PJ, Burbach G, Heinzerling LM Edenharter G, Bachert C, Bindslev-Jensen C, et al. GA2LEN skin test study III: minimum battery of test inhalent allergens needed in epidemiological studies in patients. Allergy. 2009;64(11):1656-62.

26. Nathan RA, Sorkness CA, Kosinski M, Schatz M, Li JT, Marcus P, et al. Development of the asthma control test: a survey for assessing asthma control. J Allergy Clin Immunol. 2004;113(1):59-65.

27. Kowalski ML. Heterogeneity of NSAIDExacerbated Respiratory Disease: has the time come for subphenotyping? Curr Opin Pulm Med. 2019;25(1):64-70.

28. Schulman ES. Development of a monoclonal anti-immunoglobulin E antibody (omalizumab) for the treatment of allergic respiratory disorders. Am J Respir Crit Care Med. 2001;164(8 Pt 2):S6-11.

29. Zhang N, Holtappels G, Gevaert P, Patou J, Dhaliwal B, Gould H, et al. Mucosal tissue polyclonal IgE is functional in response to allergen and SEB. Allergy. 2011;66(1):141-8.

30. Szczeklik A, Nizankowska E, Duplaga M. Natural history of aspirin-induced asthma. AIANE Investigators. European Network on Aspirin-Induced Asthma. Eur Respir J. 2000;16(3):432-6.

31. MacDonald KM, Kavati A, Ortiz B, Alhossan A, Lee CS, Abraham I. Short- and long-term real-world effectiveness of omalizumab in severe allergic asthma: systematic review of 42 studies published 2008-2018. Expert Rev Clin Immunol. 2019;15(5):553-69.

32. Bidder T, Sahota J, Rennie C, Lund VJ, Robinson DS, Kariyawasam HH. Omalizumab treats chronic rhinosinusitis with nasal polyps and asthma together-a real life study. Rhinology. 2018;56(1):42-5

33. Tiotiu A, Oster JP, Roux P, Nguyen Thi PL, Peiffer $G$, Bonniaud $P$, et al. Omalizumab's effectiveness in severe allergic asthma and nasal polyps: a real-life study. J Investig Allergol Clin Immunol. 2020:30,1

34. Porcaro F, Di Marco A, Cutrera R Omalizumab in patient with aspirin exacerbated respiratory disease and chronic idiopathic urticaria. Pediatr Pulmonol. 2017;52(5):E26-E8.

35. Tomassen P, Vandeplas G, Van Zele T, Cardell LO, Arebro J, Olze $\mathrm{H}$, et al. Inflammatory endotypes of chronic rhinosinusitis based on cluster analysis of biomarkers. J Allergy Clin Immunol. 2016;137(5):1449-56 e4.

36. Fokkens WJ, Lund V, Bachert C, Mullol J, Bjermer L, Bousquet J, et al. EUFOREA consensus on biologics for CRSWNP with or without asthma. Allergy. 2019. 74(12): 23122319.

37. Bachert C, Han JK, Desrosiers M, Hellings PW, Amin N, Lee SE, et al. Efficacy and safety of dupilumab in patients with severe chronic rhinosinusitis with nasal polyps (LIBERTY NP SINUS-24 and LIBERTY NP SINUS-52): results from two multicentre, randomised, double-blind, placebo-controlled, parallel-group phase 3 trials. Lancet. 2019. 394 (10209): 1638-1650.

38. Laidlaw TM, Mullol J, Fan C, Zhang D, Amin N, Khan A, et al. Dupilumab improves nasal polyp burden and asthma control in patients with CRSWNP and AERD. J Allergy Clin Immunol Pract. 2019;7(7):2462-5

Ulrike Förster-Ruhrmann MD Department of Oto-Rhino-Laryngolo-

gy, Campus Virchow-Klinikum

Charité-Universitätsmedizin Berlin

Augustenburger Platz 1

13353 Berlin

Tel: +49 30-450-555002

fax: +49 30-450-555900

E-mail ulrike.foerster@charite.de 